RIBEIRO, L.G.; RODRIGUES, C.; EDSON FILHO. F. Avaliação de cultivares de alface no plantio de primavera-verão em Alegre-ES Horticultura Brasileira, Brasília, v. 10, n. 1, p. 41, 1992.

ROSA, J.; PEIL, R.M.; MARTINS, S.R.; MENDES, M.E.; SCHWENGBER, J.E. Avaliação de cultivares de alface em estufa plástica no inverno no sul do Rio Grande do Sul. Horticultura Brasileira, Brasília, v. 14, n. 1, p. 113, 1996.

SADY, W.; ROZEK, S.; MYCZKOWSKI, J. Effect of different forms of nitrogen on the quality of lettuce yield. In: GROWING MEDIA E PLANT NUTRITION. Acta Horticulturae, v. 401, p. 409-416, 1995.
STEINER, A.A. The universal nutrient solution. In: INTERNATIONAL CONGRESS ON SOILLESS CULTURE, 6., 1984, Lunteren. Proceedings... Lunteren: International Society of Soiless Culture, 1984. p. 633-649.

SUZUKI, Y.; SHINOHARA, Y.; SHIBUYA, M.; IKEDA, H. Recent development of hydroponics in Japan. In: INTERNATIONAL CONGRESS ON SOILLESS CULTURE, 6., 1984, Lunteren. Proceedings...Lunteren: International Society of Soiless Culture, 1984. p. 661-672.

TONIOLLI, C.B.; BARROS, I.B.I. Performance de cultivares de alface durante o verão no município de Porto Alegre. Horticultura Brasileira, Brasília, v. 14, n. 1, p. 125, 1996.
WOLF, B. Improvements in azomethine-H method for determination of boron. Communications in Soil Science and Plant Analysis, v. 5, n. 1, p. 39-44, 1974.

YOKOYAMA, S.; MÜLLER, J.J.V.; SILVA, A.C.F. "EMPASC-357-litoral": nova cultivar de alface para verão. Horticultura Brasileira, Brasília, v. 9, n. 1, p. 28, 1991.

ZATARIN, M. Comportamento de progênies de alface (Lactuca sativa L.) em diferentes épocas de plantio. Piracicaba: ESALQ, 1985.90 p. (Tese mestrado).

MELLO, S.C.; VITTI, G.C. Desenvolvimento do tomateiro e modificações nas propriedades químicas do solo em função da aplicação de resíduos orgânicos, sob cultivo protegido. Horticultura Brasileira, Brasília, v. 20, n. 2, p. 200 - 206, junho 2.002.

\title{
Desenvolvimento do tomateiro e modificações nas propriedades quími- cas do solo em função da aplicação de resíduos orgânicos, sob cultivo protegido $^{1}$
}

\author{
Simone C. Mello ${ }^{2}$; Godofredo C. Vitti ${ }^{3}$ \\ ${ }^{2}$ Rua das magnólias, 135, J. Novo Mundo, 13.211-610 Jundiaí-SP; ${ }^{3}$ ESALQ - Departamento de Solos e Nutrição de Plantas, C. Postal \\ 09, 13.418-900 Piracicaba-SP; E-mail: sicmello@zaz.com.br
}

\section{RESUMO}

O experimento foi conduzido de fevereiro a agosto de 1997 no município de Piracicaba (SP), a fim de avaliar os efeitos de doses e resíduos orgânicos no desenvolvimento do tomateiro (Lycopersicon esculentum Mill) cultivado em estufa e nos atributos químicos de um solo Podzólico vermelho-amarelo abrupto, A moderado, textura arenosa/média. $\mathrm{O}$ delineamento experimental foi de blocos casualizados em esquema fatorial $3 \times 4$, sendo usados três resíduos orgânicos [composto de lodo de cervejaria e cavaco de eucalipto (CLC), composto de lixo (CL) e cama de frango (CF)] e quatro doses equivalentes a 0 ; $100 ; 150$ e $200 \mathrm{~kg} \mathrm{ha}^{-1}$ de N, com quatro repetições. A produção de frutos de tomate não aumentou com a adição dos materiais orgânicos. A aplicação de $\mathrm{CF}$ reduziu o $\mathrm{pH}$ e o teor de $\mathrm{Mg}$ no solo. As doses do CLC elevaram o conteúdo de matéria orgânica, o teor de cálcio trocável e a capacidade de troca de cátions do solo. O CL, por sua vez, não alterou as propriedades químicas do solo.

Palavras-chave: Lycopersicon esculentum, adubação orgânica, cama de frango, composto de lixo, nitrogênio.

\begin{abstract}
Development of tomato and changes in soil properties with organic materials application in greenhouse

An experiment was carried out between February and August/ 97 to evaluate the effects of different organic wastes on the growth of tomato (Lycopersicon esculentum Mill) plants and on the chemical properties of an Ultisol soil. The experimental design was of complete randomized blocks with four replicates and treatments disposed in a factorial $3 \times 4$ : three organic wastes [malt residue and eucalyptus chip compost (CLC), urban solid waste compost (CL) and chicken litter (CF)] and four rates equivalent to $0 ; 100 ; 150$ and $200 \mathrm{~kg} \mathrm{ha}^{-1}$ of $\mathrm{N}$. The fruit yield did not increase with organic waste additions. CF application decreased the $\mathrm{pH}$ and exchange $\mathrm{Mg}$ of soil. CLC addition increased the organic matter; exchange $\mathrm{Ca}$ and cation exchange capacity of soil. However, CL did not change the soil chemical properties.
\end{abstract}

Keywords: Lycopersicon esculentum, organic manure, chicken litter, urban solid waste, nitrogen.

(Aceito para publicação em 15 de fevereiro de 2.002)

\begin{abstract}
$\mathrm{C}$ om o surgimento de novos processos de tratamento que permitem a redução do potencial poluidor dos resíduos orgânicos, o uso agronômico deles, como fonte de nutrientes às plantas e como condicionadores dos solos, tem se constituído em alternativa interessante na preservação da qualidade ambiental.
\end{abstract}

Além de possuírem matéria orgânica, que pode melhorar as propriedades físicas, químicas e biológicas do solo, os materiais orgânicos contêm nutrientes, dentre eles o N. Para espécies olerícolas, como o tomateiro, que apresenta alto requerimento por nutrientes em tempo relativamente curto, os materiais orgânicos são normalmente em- pregados visando suprir parte da necessidade de $\mathrm{N}$ pela planta, uma vez que a liberação desse nutriente depende da taxa de mineralização do material orgânico. Gerber et al. (1981) obtiveram aumento de $25,5 \%$ na produção de tomate com a aplicação de lodo de esgoto em quantidade equivalente a $112 \mathrm{~kg} \mathrm{ha}^{-1}$ de $\mathrm{N}$, em relação à adição de 112-67-67

\footnotetext{
${ }^{1}$ Parte da Tese de Doutorado apresentada ao Departamento de Solos e nutrição de plantas da ESALQ/USP, pelo primeiro autor.
} 
$\mathrm{kg} \mathrm{ha} \mathrm{h}^{-1}$ de $\mathrm{N}, \mathrm{P}_{2} \mathrm{O}_{5}$ e $\mathrm{K}_{2} \mathrm{O}$ como fertilizante mineral. Aumento no peso de matéria fresca de plantas de tomate, após sete semanas do transplante das mudas, foi constatado com as aplicações de 2 a $10 \%$, em peso seco, de lodo de esgoto, atribuído à presença de $\mathrm{N}$ no material (Elliott \& Singer, 1988). Em um Regossolo de textura arenosa, a produção média de oito anos de cultivo de tomate foi aproximadamente $85 \%$ superior com a aplicação de $56 \mathrm{~kg} \mathrm{ha}^{-1}$ de $\mathrm{N}$, $84 \mathrm{~kg} \mathrm{ha}^{-1}$ de $\mathrm{P}_{2} \mathrm{O}_{5}$ e $56 \mathrm{~kg} \mathrm{ha}^{-1}$ de $\mathrm{K}_{2} \mathrm{O}$, juntamente com $10 \mathrm{t}^{-1}$ de esterco de curral, em relação à adubação mineral, cuja produção foi de 14,31 tha ${ }^{-1}$ (Freitas \& Faria, 1981).

Quanto aos benefícios gerados pela adição de materiais orgânicos nas propriedades químicas do solo, são relevantes o aumento do $\mathrm{pH}$, da capacidade de troca de cátions (CTC) e dos teores de nutrientes no solo. No pH do solo, Mazur et al. (1983) obtiveram elevação de 5,2 para 5,7 com a aplicação de $30 \mathrm{t}$ ha ${ }^{-1}$ de composto de resíduo urbano. Asiegbu \& Oikeh (1995) observaram aumento no $\mathrm{pH}$ do solo, após 52 dias das adições de 10; 20 e 30 t ha $^{-1}$ de esterco de coelho ou de galinha. Incrementos na CTC do solo foram obtidos com 40 e 60 t ha $^{-1}$ de adubo orgânico, proveniente de restos de feno com esterco de curral (Ndayegamiye \& Côté, 1989). Melo et al. (1994) observaram elevação na CTC quando $32 \mathrm{t} \mathrm{ha}^{-1}$ de lodo de esgoto foram aplicados ao solo. Elevações nos teores de $\mathrm{P}$ e K do solo foram constatados após a aplicação de $12 \mathrm{tha}^{-1} \mathrm{de}$ esterco de galinha (Holanda et al., 1982).

Porém, em virtude da grande diversidade de materiais orgânicos, os efeitos de suas aplicações no solo são variáveis, necessitando de novas pesquisas, visando principalmente aqueles pouco estudados, dentre os quais pode-se citar os materiais derivados do lodo biológico produzido pelas indústrias de cervejarias. Esse resíduo, produzido em grande volume, é um grande problema para as indústrias, uma vez que a sua disposição em aterros sanitários está se tornando inviável, por razões econômicas e ecológicas.

Portanto, o trabalho objetivou avaliar os efeitos da aplicação de composto de lodo de cervejaria e cavaco de eucalipto, composto de lixo e cama de frango no desenvolvimento do tomateiro e nas propriedades químicas do solo, em ambiente protegido.

\section{MATERIAL E MÉTODOS}

O experimento foi conduzido de fevereiro a agosto de 1997, em solo Podzólico vermelho-amarelo abrupto, A moderado, textura arenosa/média, cultivado com tomate cv. Carmem, em condições de estufa, no município de Piracicaba, SP.

Os resíduos orgânicos utilizados no experimento foram: 1 . composto de lodo de cervejaria e cavaco de eucalipto, proveniente da unidade de Guarulhos (SP), das Cervejarias Reunidas Skol Caracú $\mathrm{S} / \mathrm{A}$, constituído da mistura de lodo e terra infusória e cavacos de eucalipto, na proporção de 1:2, respectivamente. Este material foi compostado por cerca de 20 dias em pátio a céu aberto, sob condições de aerobiose, em sistema de leiras estáticas, com aeração forçada, cobertas com plástico. Após este período, o composto orgânico final foi separado do material não decomposto totalmente, por peneiramento; 2. composto de lixo preparado na usina de tratamento de Santo André (SP), por meio da permanência em biodigestor por 60 horas. Após esta etapa, o material foi disposto em leiras a céu aberto, revolvidos com o auxílio de pá carregadeira a cada oito dias, a fim de finalizar o processo de decomposição e peneirado em malha de $11 \mathrm{~mm}$ e; 3 . cama de frango, constituída da mistura de serragem de pinus com esterco de frango, depositado durante 45 dias. Amostras representativas de cada resíduo foram coletadas e analisadas (Tabela 1).

O solo da área experimental $\left(840 \mathrm{~m}^{2}\right)$ foi amostrado 30 dias antes do plantio, coletando-se 15 sub-amostras à profundidade de 0-20 cm, para formar uma amostra composta, e analisado no Lab. de Análise de Solos do Depto. de Solos e Nutrição de Plantas da ESALQ/USP. Os resultados foram: $\mathrm{pH}\left(\mathrm{CaCl}_{2}\right)=5,1$; matéria orgânica $=17 \mathrm{~g} \mathrm{dm}^{-3} ; \mathrm{P}$ (resina) $=64 \mathrm{mg} \mathrm{dm}^{-3} ; \mathrm{K}, \mathrm{Ca}$ e $\mathrm{Mg}=4,6,52$ e 23 mmol $_{\mathrm{c}} \mathrm{dm}^{-3}$, respectivamente; $\mathrm{H}+\mathrm{Al}=$ $15 \mathrm{mmol}_{\mathrm{c}} \mathrm{dm}^{-3} ; \mathrm{CTC}=94,6 \mathrm{mmol}_{\mathrm{c}} \mathrm{dm}^{-3}$ e saturação por bases $(\mathrm{V})=84 \%$. Na adubação de plantio empregaram-se 300 $\mathrm{kg} \mathrm{ha}{ }^{-1}$ de $\mathrm{P}_{2} \mathrm{O}_{5}$ como superfosfato simples $\left(18 \%\right.$ de $\left.\mathrm{P}_{2} \mathrm{O}_{5}\right)$ em área total da estufa, segundo Trani et al. (1996). Os materiais orgânicos foram aplicados juntamente com o fósforo. $\mathrm{O}$ nitrogênio e o potássio, em cobertura, foram fornecidos, durante o ciclo da cultura, via fertirrigação, totalizando $380 \mathrm{~kg} \mathrm{ha}^{-1}$ de $\mathrm{N}$ e $450 \mathrm{~kg} \mathrm{ha}^{-1}$ de $\mathrm{K}_{2} \mathrm{O}$, conforme indicações de Vivancos (1993), empregando-se as fontes nitrato de potássio (13\% de $\mathrm{Ne} 44 \%$ de $\mathrm{K}_{2} \mathrm{O}$ ) e uréia ( $45 \%$ de $\left.\mathrm{N}\right)$.

As mudas de tomate cv. Carmem foram produzidas em bandejas, em casa-devegetação, e transplantadas para uma estufa do tipo arco, coberta com filme de polietileno aditivado, no espaçamento de $0,5 \mathrm{~m}$ entre plantas e 1,0 $\mathrm{m}$ entre fileiras, sendo que as plantas foram conduzidas em fitilhos. Pulverizações preventivas à podridão apical foram feitas semanalmente, a partir do início do florescimento, dirigidas aos frutos, com uma solução de cloreto de cálcio a $0,6 \%$ e espalhante adesivo (Castellane, 1988). Para o controle de pragas e doenças foram utilizados inseticidas e fungicidas cúpricos, respectivamente, conforme as necessidades da cultura.

O delineamento experimental foi de blocos casualizados com quatro repetições, no esquema fatorial $3 \times 4$, constituído de três resíduos orgânicos (composto de lodo de cervejaria e cavaco de eucalipto; composto de lixo e cama de frango) e quatro doses de $\mathrm{N}(0 ; 100 ; 150$ e $200 \mathrm{~kg} \mathrm{ha}^{-1}$ ), tendo como fontes os resíduos orgânicos. Essas quantidades de $\mathrm{N}$ foram equivalentes a: $0 ; 7,0 ; 10,5 \mathrm{e}$ $14 \mathrm{t} \mathrm{ha}^{-1}$ de composto de lodo de cervejaria + cavo de eucalipto (CLC); 0; 2,9; 4,4 e $5,9 \mathrm{t} \mathrm{ha}^{-1}$ de cama de frango (CF); $0 ; 8,2 ; 12,3$ e 16,4 t ha $^{-1}$ de composto de lixo (CL). Cada parcela foi composta por 16 plantas com plantio em linha dupla, ou seja, oito plantas por linha, totalizando uma área de $8,0 \mathrm{~m}^{2}$.

Para as determinações dos teores totais de macro e micronutrientes, na época do florescimento, foram coletadas cinco folhas por parcela, tomando-se a quarta folha a partir do ápice da planta, conforme metodologia descrita por Malavolta et al. (1997).

A colheita dos frutos ocorreu durante 56 dias, iniciando-se em 12/05 e se estendendo até 07/07/97. A produção to- 
Tabela 1. Propriedades químicas, em base seca, dos materiais orgânicos empregados. Piracicaba, ESALQ, 1997.

\begin{tabular}{|c|c|c|c|}
\hline Propriedade química & CLC $^{1}$ & $\mathrm{CL}^{2}$ & $\mathrm{CF}^{3}$ \\
\hline $\mathrm{pH}$ em $\mathrm{CaCl}_{2} 0,01 \mathrm{M}$ & 7,80 & 8,30 & 8,20 \\
\hline Matéria orgânica total $\left(\mathrm{g} \mathrm{kg}^{-1}\right)$ & 500,45 & 692,90 & 862,75 \\
\hline Matéria orgânica compostável $\left(\mathrm{g} \mathrm{kg}^{-1}\right)$ & 479,93 & 496,06 & 713,20 \\
\hline Carbono total $\left(\mathrm{g} \mathrm{kg}^{-1}\right)^{4}$ & 277,88 & 385,01 & 479,32 \\
\hline Carbono orgânico $\left(\mathrm{g} \mathrm{kg}^{-1}\right)$ & 266,73 & 275,54 & 396,22 \\
\hline Resíduo mineral total ( $\left.\mathrm{g} \mathrm{kg}^{-1}\right)$ & 499,55 & 262,72 & 137,25 \\
\hline Resíduo mineral insolúvel $\left(\mathrm{g} \mathrm{kg}^{-1}\right)$ & 418,82 & 156,80 & 7,83 \\
\hline Resíduo mineral solúvel $\left(\mathrm{g} \mathrm{kg}^{-1}\right)$ & 80,73 & 105,92 & 129,42 \\
\hline$N$ total $\left(\mathrm{g} \mathrm{kg}^{-1}\right)^{5}$ & 14,27 & 12,23 & 33,78 \\
\hline$P$ total $\left(\mathrm{g} \mathrm{kg}^{-1}\right)^{6}$ & 2,92 & 1,72 & 9,98 \\
\hline $\mathrm{K}$ total $\left(\mathrm{g} \mathrm{kg}^{-1}\right)^{7}$ & 1,11 & 3,60 & 27,00 \\
\hline Ca total $\left(\mathrm{g} \mathrm{kg}^{-1}\right)^{7}$ & 55,31 & 14,99 & 21,24 \\
\hline Mg total $\left(\mathrm{g} \mathrm{kg}^{-1}\right)^{7}$ & 1,78 & 7,69 & 5,34 \\
\hline$S$ total $\left(\mathrm{g} \mathrm{kg}^{-1}\right)^{8}$ & 1,34 & 2,56 & 2,36 \\
\hline Cu total $\left(\mathrm{mg} \mathrm{kg}^{-1}\right)^{9}$ & 111,51 & 197,24 & 186,31 \\
\hline Mn total $\left(\mathrm{mg} \mathrm{kg}^{-1}\right)^{9}$ & 535,24 & 108,48 & 322,94 \\
\hline $\mathrm{Zn}$ total $\left(\mathrm{mg} \mathrm{kg}^{-1}\right)^{9}$ & 156,11 & 364,89 & 365,17 \\
\hline Fe total $\left(\mathrm{mgkg}^{-1}\right)^{9}$ & 18443,35 & 10927,02 & 787,48 \\
\hline $\mathrm{Na}$ total $\left(\mathrm{mg} \mathrm{kg}^{-1}\right)$ & 3822,48 & 3909,27 & 4193,27 \\
\hline Relação C/N (C total e N total) & $19 / 1$ & $31 / 1$ & $14 / 1$ \\
\hline
\end{tabular}

1/ CLC: composto de lodo de cervejaria e cavaco de eucalipto; ${ }^{2} / \mathrm{CL}$ : composto de lixo; ${ }^{3 / \mathrm{CF}}$ : cama de frango; ${ }^{4 / \mathrm{C}}$ total (oxidação da matéria orgânica com solução $0,17 \mathrm{~mol} \mathrm{~L}^{-1}$ de dicromato de potássio e titulação do excesso de dicromato com solução de sulfato ferroso amoniacal $\left.0,5 \mathrm{~mol} \mathrm{~L}^{-1}\right) ;{ }^{5} / \mathrm{N}$ (método micro-kjeldhal); ${ }^{6} / \mathrm{P}$ (método do vanadato-molibdato); ${ }^{7} / \mathrm{K}, \mathrm{Ca}$ e $\mathrm{Mg}$ (espectrofotometria de chama); ${ }^{8} / \mathrm{S}$ (método gravimétrico); ${ }^{9} / \mathrm{Cu}, \mathrm{Mn}, \mathrm{Zn}$ e Fe (espectrofotometria de absorção atômica).

tal e a produção de frutos comercializáveis (frutos com diâmetro maior que $5,5 \mathrm{~cm}$ ), bem como o peso médio de frutos e o número de frutos por planta foram avaliados nas 16 plantas da parcela.

No final do experimento, em 06/08/ 97 , foram coletadas amostras de solo (20 amostras simples/parcela), para as determinações do $\mathrm{pH}\left(\mathrm{em} \mathrm{CaCl}_{2}\right)$, matéria orgânica (método colorimétrico), fósforo disponível (extraído por resina trocadora de íons e determinado por método colorimétrico), potássio, cálcio e magnésio (extraídos por resina trocadora de íons e determinados por espectrofotometria de absorção atômica) e acidez potencial (método tampão SMP). A capacidade de troca de cátions foi calculada pela soma das bases $\left(\mathrm{K}^{+}, \mathrm{Ca}^{++}\right.$e $\left.\mathrm{Mg}^{++}\right)$e da acidez potencial $\left(\mathrm{H}^{+}+\mathrm{Al}^{3+}\right)$.

Os resultados obtidos foram submetidos à análise de variância pelo teste $\mathrm{F}$, a 5\% de significância, com comparação entre médias feita pelo teste de Tukey para os resíduos orgânicos e regressão polinomial para as doses.

\section{RESULTADOS E DISCUSSÃO}

Para o número de frutos por planta $(39,3)$, peso médio de frutos $(122,6 \mathrm{~g})$, produção total $\left(108,9 \mathrm{t} \mathrm{ha}^{-1}\right) \mathrm{e}$ de frutos comercializáveis $\left(101,7 \mathrm{t} \mathrm{ha}^{-1}\right)$, a análise de variância não acusou diferença significativa para a interação dos fatores materiais orgânicos e doses, assim como dos fatores isoladamente. Para as doses, a análise de regressão polinomial também não foi significativa. Esses resultados indicam que a adubação mineral com N, aplicada via fertirrigação, foi suficiente para o desenvolvimento da cultura, embora existam indicações de resposta do tomateiro, em cultivo protegido, com a adição de até $540 \mathrm{~kg} \mathrm{ha}^{-1}$ de N. Por sua vez, Hampton et al. (1994) também não observaram incrementos na produção de tomate com a aplicação de 16 a $48 \mathrm{t} \mathrm{ha}^{-1}$ de materiais derivados de lixo sólido, em solo adubado com 240 $\mathrm{kg} \mathrm{ha}^{-1}$ de $\mathrm{N}$, atribuindo esse resultado às baixas doses adicionadas ou ao fertilizante inorgânico aplicado em todos os tratamentos.

Os teores foliares de macronutrientes e de micronutrientes não variaram entre os materiais orgânicos dentro de cada dose. As concentrações médias, em $\mathrm{g} \mathrm{kg}^{-1}$, de $\mathrm{N}, \mathrm{P}, \mathrm{K}, \mathrm{Ca}$, Mg e S foram: 43,2; 7,1; 31,9; 22,5; 6,4 e 5,3. Para o B, Cu, Fe, Mn e Zn foram encontrados os seguintes teores foliares médios, em mg kg-1: 56,9; 212,5; 108,7; 91,2 e 30,6. Dessa forma, as diferenças entre os conteúdos de nutrientes nos materiais orgânicos não refletiram na concentração foliar.

Os teores foliares de $\mathrm{K}$ aumentaram linearmente com a aplicação do CLC (Figura 1), provavelmente devido ao fornecimento ao solo desse elemento 
pelo material orgânico, que se tornou disponível para as plantas. Resultados concordantes foram obtidos por Asiegbu \& Oikeh (1995), os quais observaram acréscimos nos teores foliares de $\mathrm{K}$ após a adição de esterco de suíno ou lodo de esgoto, em quantidades que variaram de 10 a 30 t ha $^{-1}$. Por outro lado, as análises de regressões não foram significativas para os teores de $\mathrm{K}$ nas folhas com a aplicação do CL e da CF.

A adição do CLC diminuiu os teores de enxofre nas folhas (Figura 1). Essa redução, entretanto, foi pequena, não prejudicando o desenvolvimento da planta. As aplicações do CL reduziram linearmente as concentrações de Fe e Mn nas folhas (Figura 2). Provavelmente, as quantidades de matéria orgânica $\left(8,2\right.$ a $\left.16,4 \mathrm{tha}^{-1}\right)$ adicionadas por esse composto, bem como suas propriedades químicas (Tabela 1) reduziram a disponibilidade de $\mathrm{Fe}$ e $\mathrm{Mn}$ através da complexação pelos compostos orgânicos, o que resultou em menor acúmulo desses metais no tecido vegetal. Mugwira (1979) também constatou redução nas concentrações de Fe e Mn em plantas de milheto, com o aumento das doses de esterco de curral, devido, neste caso, ao aumento do $\mathrm{pH}$ proporcionado pelo adubo.

Para os teores foliares de $\mathrm{Cu}$, ajustaram-se equações de regressões quadráticas em função da aplicação de CL (Figura 2) e CF, sendo que para este material orgânico a equação de regressão foi a seguinte: $\mathrm{y}=0,0046 \mathrm{x}^{2}-1,0433 \mathrm{x}$ $+247,05\left(\mathrm{R}^{2}=0,99\right)$. Fritz \& Venter (1988) verificaram o mesmo comportamento em frutos de tomate, em que a adição de $50 \mathrm{t} \mathrm{ha}^{-1}$ de composto de lixo reduziu o conteúdo de $\mathrm{Cu}$, que aumentou posteriormente com a aplicação de $100 \mathrm{t} \mathrm{ha}^{-1}$.

Com exceção do $\mathrm{Cu}$, os teores dos nutrientes encontram-se dentro da faixa considerada adequada, segundo Trani \& Raij (1996). Os teores foliares de N, $\mathrm{K}$ e Mg também foram semelhantes aos obtidos por Asiegbu \& Oikeh (1995). Os teores de $\mathrm{Cu}$, apesar de elevados, provavelmente devido à contaminação por pulverizações foliares com fungicidas cúpricos, não foram tóxicos às plantas.

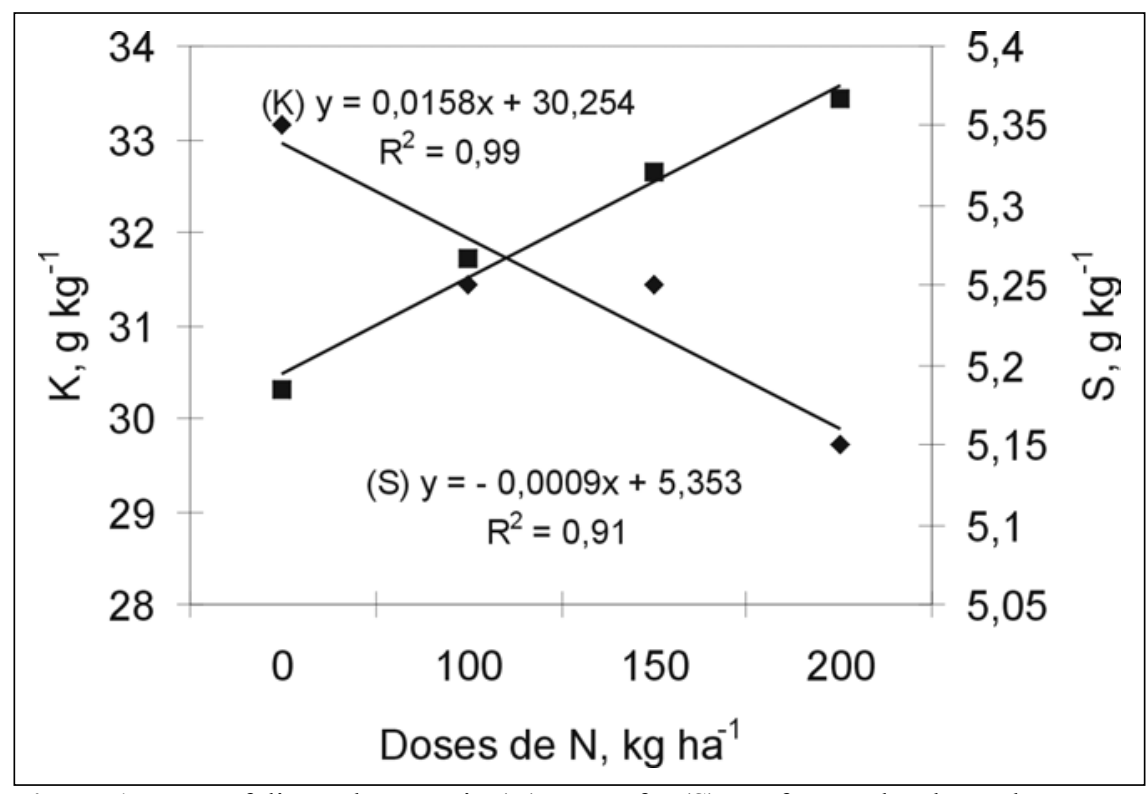

Figura 1. Teores foliares de potássio (K) e enxofre (S) em função das doses de $\mathrm{N}$ como composto de lodo de cervejaria e cavaco de eucalipto. (Média de quatro repetições). Piracicaba, ESALQ, 1997.

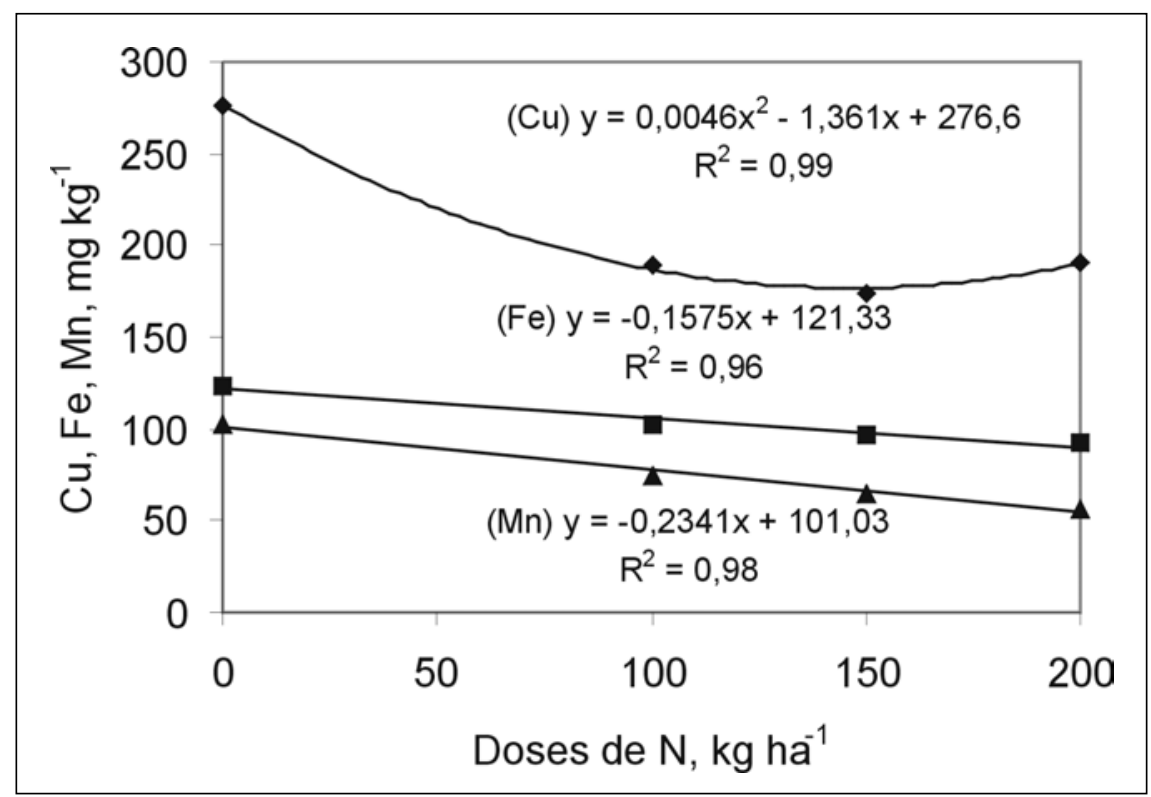

Figura 2. Teores foliares de cobre $(\mathrm{Cu})$, ferro $(\mathrm{Fe})$ e manganês $(\mathrm{Mn})$ em função das doses de $\mathrm{N}$ como composto de lixo. (Média de quatro repetições). Piracicaba, ESALQ, 1997.

As propriedades químicas do solo, aos seis meses após a incorporação dos materiais orgânicos, constam da Tabela 2. Com exceção dos teores de Ca trocáveis e da CTC, as características do solo não foram alteradas pelo tipo de material orgânico. Nas doses mais elevadas, os teores de Ca trocáveis foram superiores para o CLC em relação aos demais, em virtude da maior quantidade de $\mathrm{Ca}$ encontrada nesse material (Tabela 1). O mesmo comportamento foi observado para a CTC do solo, que foi maior para o CLC, provavelmente devido à natureza química desse material.

$\mathrm{O}$ pH sofreu redução linear com a adição da CF (Figura 3), possivelmente devido aos processos de nitrificação do amônio e produção de ácidos orgânicos, resultantes do ataque ao resíduo por fungos decompositores, o que está de acordo com as observações de Chang et al. (1991). Estes autores obtiveram reduções no $\mathrm{pH}$ de 0,3 a 0,7 unidade após onze 
Tabela 2. Propriedades químicas do solo, em função do material orgânico e dose de nitrogênio. Piracicaba, ESALQ, 1997.

\begin{tabular}{|c|c|c|c|c|c|}
\hline \multirow{2}{*}{$\begin{array}{l}\text { Materiais } \\
\text { orgânicos }\end{array}$} & \multicolumn{4}{|c|}{ Doses de N (kg ha $\left.{ }^{-1}\right)$} & \multirow{2}{*}{ Teste $F^{4}(\%)$} \\
\hline & 0 & 100 & 150 & 200 & \\
\hline \multicolumn{6}{|c|}{$\mathrm{pH}\left(\mathrm{CaCl}_{2}\right)$} \\
\hline $\mathrm{CLC}^{1}$ & $5,75 \mathrm{a}$ & $5,73 \mathrm{a}$ & $6,03 \mathrm{a}$ & $5,93 \mathrm{a}$ & ns \\
\hline $\mathrm{CF}^{2}$ & $5,83 a$ & $5,73 a$ & $5,70 a$ & $5,58 a$ & $3,32(\mathrm{~L})$ \\
\hline $\mathrm{CL}^{3}$ & 5,78 a & $5,60 \mathrm{a}$ & $5,80 \mathrm{a}$ & $5,75 a$ & ns \\
\hline C.V. (\%) & 3,65 & & & & \\
\hline \multicolumn{6}{|c|}{ Matéria orgânica $\left(\mathrm{g} \mathrm{dm}^{-3}\right)$} \\
\hline$\overline{C L C}$ & $16,75 \mathrm{a}$ & $18,50 \mathrm{a}$ & $19,25 \mathrm{a}$ & $21,25 a$ & $2,48(L)$ \\
\hline CF & $21,00 \mathrm{a}$ & $21,50 \mathrm{a}$ & $18,50 \mathrm{a}$ & $20,00 \mathrm{a}$ & ns \\
\hline $\mathrm{CL}$ & $21,25 \mathrm{a}$ & $17,25 \mathrm{a}$ & $16,25 \mathrm{a}$ & $17,50 \mathrm{a}$ & ns \\
\hline C.V. (\%) & 19,07 & & & & \\
\hline \multicolumn{6}{|c|}{ Fósforo $\left(\mathrm{mg} \mathrm{dm}^{-3}\right)$} \\
\hline CLC & $121,50 \mathrm{a}$ & $171,50 \mathrm{a}$ & $198,00 \mathrm{a}$ & $165,50 \mathrm{a}$ & ns \\
\hline CF & 86,25 a & 134,75 a & 157,44 a & $136,00 \mathrm{a}$ & ns \\
\hline $\mathrm{CL}$ & $127,50 \mathrm{a}$ & 106,25 a & 233,25 a & $172,00 \mathrm{a}$ & ns \\
\hline C.V. (\%) & 31,24 & & & & \\
\hline \multicolumn{6}{|c|}{ Potássio $\left(\mathrm{mmol}_{\mathrm{c}} \mathrm{dm}^{-3}\right)$} \\
\hline$\overline{C L C}$ & $1,90 \mathrm{a}$ & $1,78 \mathrm{a}$ & $2,68 \mathrm{a}$ & $2,18 \mathrm{a}$ & ns \\
\hline CF & $2,18 \mathrm{a}$ & $2,18 \mathrm{a}$ & $2,33 \mathrm{a}$ & $1,88 \mathrm{a}$ & ns \\
\hline $\mathrm{CL}$ & $1,82 \mathrm{a}$ & $1,78 \mathrm{a}$ & $2,03 \mathrm{a}$ & $2,28 \mathrm{a}$ & ns \\
\hline C.V. (\%) & 23,31 & & & & \\
\hline \multicolumn{6}{|c|}{ Cálcio $\left(\mathrm{mmol}_{\mathrm{c}} \mathrm{dm}^{-3}\right)$} \\
\hline$\overline{C L C}$ & 49,36 a & $55,25 \mathrm{a}$ & $71,25 \mathrm{a}$ & $77,75 \mathrm{a}$ & $5,20(\mathrm{~L})$ \\
\hline $\mathrm{CF}$ & $56,00 \mathrm{a}$ & $52,00 \mathrm{a}$ & $52,75 \quad b$ & $46,00 \mathrm{~b}$ & ns \\
\hline $\mathrm{CL}$ & $47,75 \mathrm{a}$ & $42,25 a$ & $55,50 \quad b$ & $52,75 \quad b$ & ns \\
\hline C.V. $(\%)$ & 12,64 & & & & \\
\hline \multicolumn{6}{|c|}{ Magnésio $\left(\mathrm{mmol}_{\mathrm{c}} \mathrm{dm}^{-3}\right)$} \\
\hline$\overline{C L C}$ & $15,46 \quad b$ & $16,65 \mathrm{a}$ & 26,87 a & $22,27 \mathrm{a}$ & ns \\
\hline CF & $25,11 \mathrm{a}$ & $21,32 a$ & $20,08 a$ & $15,46 a$ & $3,31(L)$ \\
\hline $\mathrm{CL}$ & $18,32 a b$ & $13,59 \mathrm{a}$ & $19,63 \mathrm{a}$ & $18,89 \mathrm{a}$ & ns \\
\hline$\overline{\text { C.V. (\%) }}$ & 30,62 & & & & \\
\hline \multicolumn{6}{|c|}{ CTC $\left(\mathrm{mmol}_{\mathrm{c}} \mathrm{dm}^{-3}\right)$} \\
\hline$\overline{C L C}$ & $77,65 \quad b$ & $88,53 \mathrm{a}$ & $115,43 a$ & 116,18 a & $6,42(\mathrm{~L})$ \\
\hline $\mathrm{CF}$ & $98,93 \mathrm{a}$ & 91,93 a & $93,08 \quad b$ & $80,13 \quad b$ & ns \\
\hline $\mathrm{CL}$ & $84,33 a b$ & 74,78 a & $92,78 \quad b$ & $89,53 \mathrm{~b}$ & ns \\
\hline C.V. (\%) & 14,22 & & & & \\
\hline
\end{tabular}

*/ As diferenças entre as médias, nas colunas, não foram significativas a $5 \%$ pelo teste de Tukey;

1/ CLC: composto de lodo de cervejaria e cavaco de eucalipto; ${ }^{2 /} \mathrm{CL}$ : composto de lixo;

3/ CF: cama de frango; ${ }^{4}$ Significância do teste F para as doses de N estudadas (modelo linear - L).

aplicações anuais de 30 a $90 \mathrm{t} \mathrm{ha}^{-1}$ de esterco de curral. As aplicações dos demais materiais orgânicos não alteraram o pH do solo. Isto indica, que dependendo do tipo de resíduo aplicado pode ou não ocorrer mudanças na acidez do solo, fato tam- bém comprovado por Eira \& Carvalho (1970), que observaram aumento do $\mathrm{pH}$ com a adição de palha de cana e torta de filtro e nenhuma variação do $\mathrm{pH}$ com a adição de um composto orgânico. Os teores de $\mathrm{Mg}$ no solo foram reduzidos com a adição da CF (Figura 3), provavelmente pela diminuição no $\mathrm{pH}$ do solo, fato também reportado por Dai et al. (1995).

A adição do CLC aumentou o conteúdo de matéria orgânica do solo (Figura 4), o que pode ser explicado pela natureza 


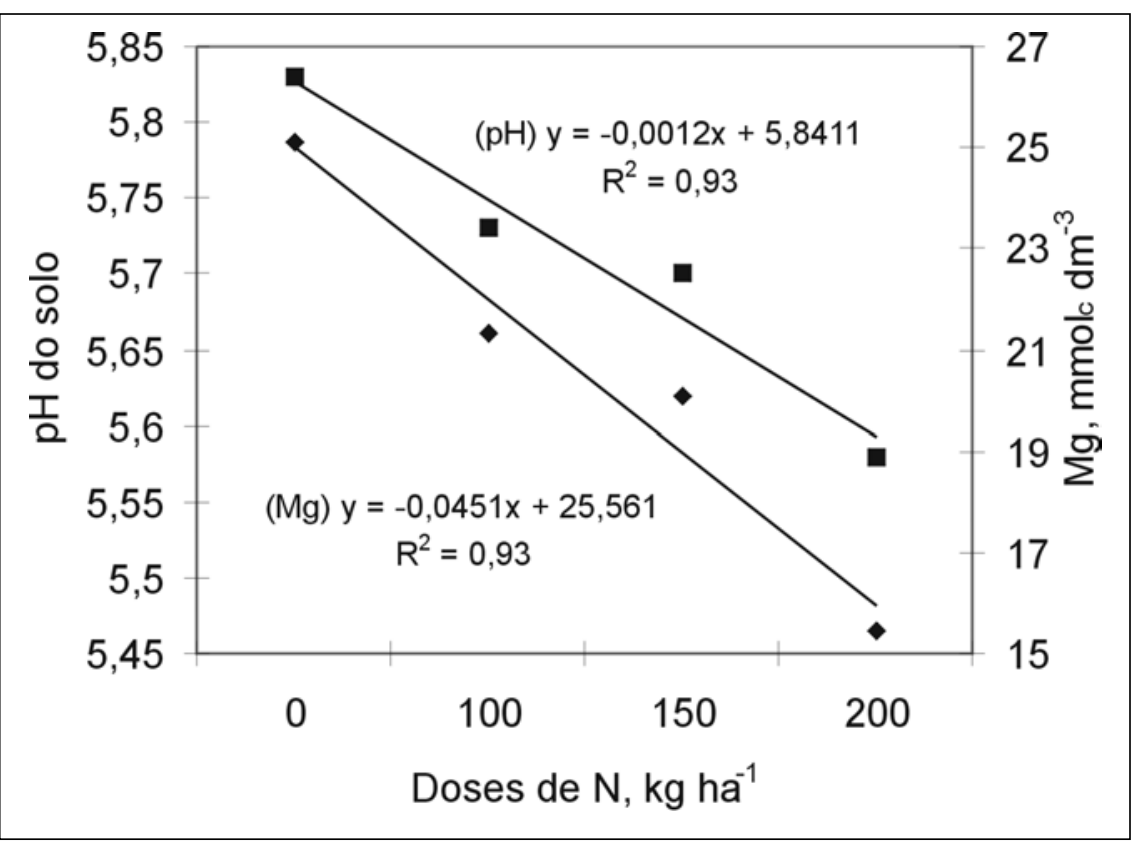

Figura 3. Valores de $\mathrm{pH}$ e teores de magnésio $(\mathrm{Mg})$ no solo em função das doses de $\mathrm{N}$ como cama de frango. (Média de quatro repetições). Piracicaba, ESALQ, 1997.

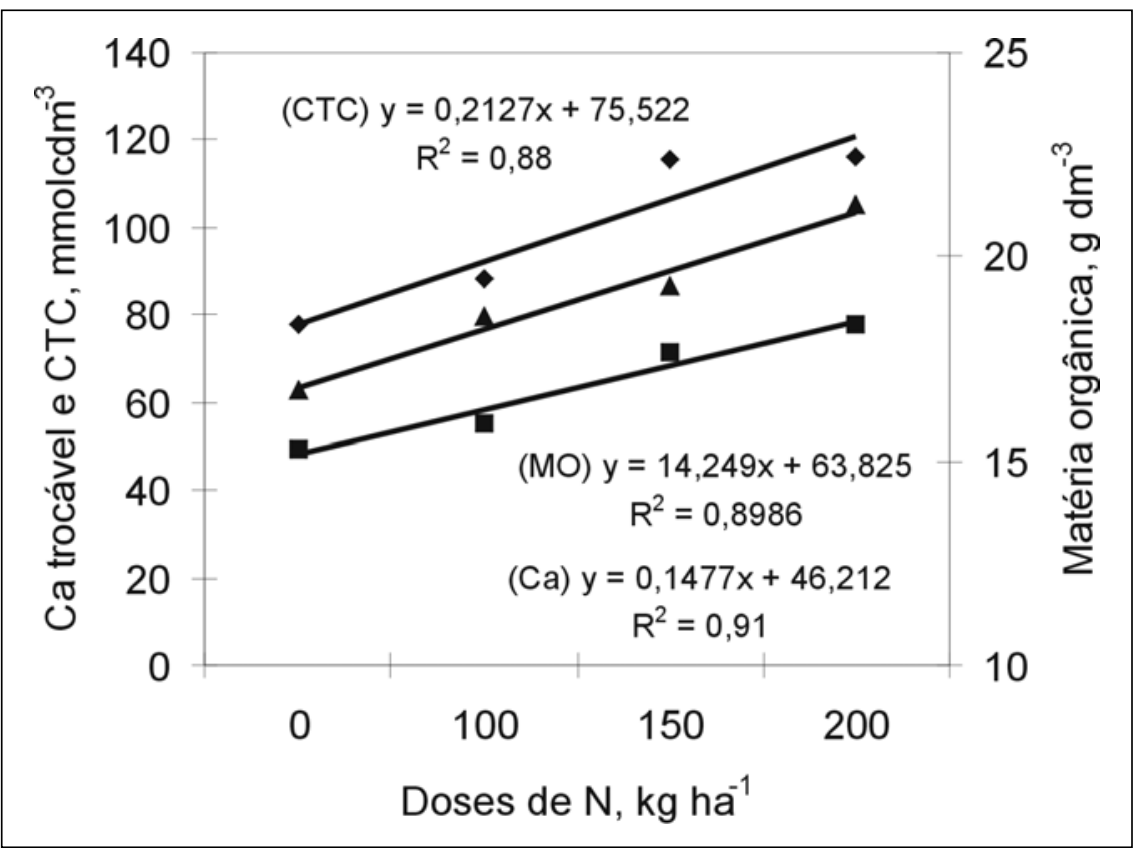

Figura 4. Teores de cálcio trocável $(\mathrm{Ca})$, capacidade de troca de cátions $(\mathrm{CTC})$ e matéria orgânica (MO) do solo em função das doses de N como composto de lodo de cervejaria e cavaco de eucalipto. (Média de quatro repetições). Piracicaba, ESALQ, 1997.

estável dos compostos orgânicos deste resíduo, mais resistentes à degradação. Incremento no conteúdo de matéria orgânica do solo também foi comprovado por Gill \& Meelu (1982), após a adição de $12 \mathrm{t} \mathrm{ha}^{-1}$ de esterco de curral misturado com restos de feno. Resultados semelhantes foram obtidos por Murillo et al. (1989), com as aplicações de 200 e teor de Ca pode ser atribuído ao fornecimento de Ca e pelo aumento da CTC. Pavan (1993) também observou aumento no teor de $\mathrm{Ca}$ do solo com a adição de esterco bovino curtido e biodigerido em dose equivalente a $120 \mathrm{~kg} \mathrm{ha}^{-1} \mathrm{de} \mathrm{N}$, com aumento concomitante da CTC do solo. A relação linear $\left(\mathrm{R}^{2}=0,88\right)$ sugere que o aumento da CTC foi causado principalmente pelo carbono orgânico contido no material. Ndayegamiye \& Côté (1989) também observaram incrementos na CTC de 7,6 e $15,2 \%$, respectivamente, com a aplicação de 40 e $60 \mathrm{t}$ ha $^{-1}$ de adubo orgânico (esterco de curral misturado com restos de feno), atribuídos aos incrementos nos conteúdos de carbono orgânico do solo.

Dessa forma, pode-se inferir que é possível melhorar a fertilidade do solo com o emprego de pequenas doses de composto de lodo de cervejaria e cavaco de eucalipto. Por outro lado, a ausência de resposta do tomateiro à adubação orgânica sugere a necessidade de novos estudos, utilizando quantidades maiores dos materiais orgânicos empregados, com o objetivo de incrementar a sua produtividade e reduzir a utilização de fertilizante mineral.

\section{LITERATURA CITADA}

ASIEGBU, J.E.; OIKEH, S. Evaluation of chemical composition of manures from different organic wastes and their potential for supply of nutrients to tomato in a tropical Ultisol. Biological Agriculture and Horticulture, v. 12, p. 47-60, 1995. CASTELLANE, P.D. Podridão apical em frutos de tomateiro. Jaboticabal: FUNEP, 1988. 39 p.

CHANG, C.; SOMMERFELDT, T.G.; ENTZ, T. Soil chemistry after eleven annual applications of cattle feedlot manure. Journal of Environmental Quality, v. 20, p. 475-480, 1991.

DAI, ZH.; LIU, YX.; WANG, XJ.; ZHAO, D.W. Acidification and change of physicochemical properties of Mt theng soils in last 34 years. Journal of Environmental Science, v. 7, p. 346351, 1995.

EIRA, A.F.; CARVALHO, P.C.T. A decomposição da matéria orgânica pelos microrganismos do solo e sua influência nas variações do $\mathrm{pH}$. Revista de Agricultura, v. 45, p. 15-21, 1970.

ELLIOTT, H.A.; SINGER, L.M. Effect of water treatment sludge on growth and elemental composition of tomato (Lycopersicon esculentum) shoots. Communications in Soil Science and Plant Analysis, v. 19, p. 345-354, 1988.

FREITAS, M.B.; FARIA, C.M.B. Influência de práticas agrícolas na fertilidade do solo e no rendimento do tomateiro no Agreste Pernambucano. Revista Brasileira de Ciência do Solo, v. 5, p. 5457, 1981.

FRITZ, D.; VENTER, F. Heavy metals in some 
vegetable crops as influenced by municipal waste composts. Acta Horticulturae, v. 222, p. 51-62, 1988.

GERBER, J.M.; SWIADER, J.M.; PECK, T.R. Sewage sludge on vegetables - A mixed Blessing. Illinois Research, v. 23, p. 12-13, 1981.

GILL, H.S.; MEELU, O.P. Studies on the substitution of inorganic fertilizers with organic manure and their effect on soil fertility in ricewheat rotation. Fertilizer Research, v. 3, p. 303314, 1982.

HAMPTON, M.O.; SCHAFFER, B.; BRYAN, H.H. Nutrient concentrations growth and yield of tomato and squash in municipal solid-waste amended soil. Hortscience, v. 29, p. 785-788, 1994

HOLANDA, J.J.; MIELNICZU, J.; STAMMEL, J.G. Utilização de esterco e adubo mineral em quatro sequências de culturas em solo de encosta basáltica do Rio Grande do Sul. Revista Brasileira de Ciência do Solo, v. 6, p. 47-51, 1982.

MALAVOLTA, E.; VITTI, G. C.; OLIVEIRA, S
A. Avaliação do estado nutricional das plantas: princípios e aplicações. Piracicaba: POTAFOS, 1997. 319 p.

MAZUR, N.; VELLOSO, A.C.X.; SANTOS, G. A. Efeito do composto de resíduo urbano no $\mathrm{pH}$ e alumínio trocável em solo ácido. Revista Brasileira de Ciência do Solo, v. 7, p. 157-159, 1983. MELO, W.J.; MARQUES, M.O.; SANTIAGO, G.; CHELLI, R.A. Efeito de doses crescentes de lodo de esgoto sobre frações da matéria orgânica e CTC de um Latossolo cultivado com cana-deaçúcar. Revista Brasileira de Ciência do Solo, v. 18, p. 449-455, 1994.

MUGWIRA, L.M. Residual effects of dairy cattle manure on millet and rye forage and soil properties. Journal of Environmental Quality, v. 8, p. 251-255, 1979.

MURILLO, J.M.; HERNANDEZ, J.M.; BARROSO, M.; LOPEZ, R. Produccion frente a contaminacion en la utilizacion agricola de compostos urbanos. Anales de Edafologia y Agrobiologia, v. 48, p. 143-160, 1989.

NDAYEGAMIYE, A.; CÔTÉ, D. Effect of long- term pig slurry and solid cattle manure application on soil chemical and biological properties. Canadian Journal of Soil Science, v. 69, p. 39-47, 1989.

PAVAN, M.A. Avaliação de esterco de bovino biodigerido e curtido na fertilidade do solo e na nutrição e produção do cafeeiro. Londrina: IAPAR, 1993. 16p. (IAPAR. Boletim Técnico, 45). TRANI, P.E.; RAIJ, B. van. Hortaliças. In: RAIJ, B. van; CANTARELLA, H.; QUAGGIO, J.A.; FURLANI, A.M.C., ed. Recomendações de adubação e calagem para o Estado de São Paulo. Campinas: Instituto Agronômico de Campinas, 1996. p. 155-203. (Boletim Técnico, 100)

TRANI, P.; NAGAI, H.; PASSOS, F.A. Tomate (estaqueado). In: RAIJ, B. van; CANTARELLA, H.; QUAGGIO, J.A.; FURLANI, A.M.C., ed. Recomendações de adubação e calagem para o Estado de São Paulo. Campinas: Instituto Agronômico de Campinas, 1996. p. 184. (Boletim Técnico, 100) VIVANCOS, A.D. Fertirrigacion. Madrid: Mundi-Prensa, 1993. 217 p.

MAROUELLI, W.A.; SILVA, W.L.C. Profundidade de instalação da linha de gotejadores em tomateiro para processamento industrial. Horticultura Brasileira, Brasília, v. 20, n. 2, p. 206-210, junho 2.002 .

\section{Profundidade de instalação da linha de gotejadores em tomateiro para processamento industrial}

Waldir A. Marouelli; Washington L.C. Silva

Embrapa Hortaliças, C. Postal 218, 70.359-970, Brasília/DF. E-mail: waldir@cnph.embrapa.br.

\section{RESUMO}

Avaliou-se a resposta do tomateiro para processamento industrial, irrigado por gotejamento, a diferentes profundidades de instalação da linha lateral de gotejadores $(0 ; 20$ e $40 \mathrm{~cm})$. Um tratamento irrigado por aspersão foi utilizado como controle. O experimento foi conduzido na Embrapa Hortaliças, nas condições edafoclimáticas da região de cerrados do Brasil Central. Maior crescimento vegetativo de plantas foi observado nos tratamentos irrigados por aspersão e gotejamento superficial, enquanto que menor crescimento ocorreu no gotejamento a $40 \mathrm{~cm}$. A produtividade comercial do tratamento irrigado por gotejamento superficial $\left(124\right.$ t.ha $\left.^{-1}\right)$ foi $32 \%$ maior que no tratamento por gotejamento subterrâneo a $40 \mathrm{~cm}, 15 \%$ maior que no tratamento irrigado por aspersão, não diferindo $(\mathrm{p}>0,05)$ do tratamento por gotejamento a $20 \mathrm{~cm}$. Incrementos de produtividade foram devidos, principalmente, ao maior número de frutos por planta, uma vez que o estande final e a massa média de frutos não foram afetados pelos tratamentos. A incidência de frutos podres na irrigação por aspersão foi 112 e $453 \%$ maior que nos tratamentos irrigados por gotejamento superficial e subterrâneo, respectivamente.

Palavras-chave: Lycopersicon esculentum, gotejamento, sistema de irrigação, gotejo subterrâneo.
ABSTRACT

\section{Drip line placement depth for processing tomatoes crop}

A field experiment was carried out at Embrapa Hortaliças under soil and climate conditions of Central Brazil "cerrado" region, aiming to evaluate the response of drip-irrigated processing tomatoes to different drip line placement depths $(0 ; 20$ and $40 \mathrm{~cm})$. A control treatment was irrigated by sprinkle system. Larger plant vegetative growth was observed for the sprinkle and surface drip treatments, while smaller growth occurred for the $40 \mathrm{~cm}$-depth drip treatment. Marketable yield of the surface drip irrigated treatment (124 t.ha $\left.{ }^{-1}\right)$ was $32 \%$ larger than the $40 \mathrm{~cm}$-depth drip treatment, $15 \%$ larger than the sprinkle treatment, but did not differ $(p>0,05)$ from the 20 $\mathrm{cm}$-depth drip treatment. Yield increments were basically due to a larger number of fruits per plant, since final stand and mean fruit mass were not affected by treatments. Rotten fruit rate for the sprinkle treatment was 112 and $453 \%$ larger than for the treatments irrigated by surface and subsurface drip, respectively.

(Aceito para publicação em 21 de fevereiro de 2.002)

A aspersão é o principal método utilizado no Brasil para a irrigação do tomateiro para processamento industrial, ocupando mais de $90 \%$ da área irrigada. Por molhar a parte aérea das plantas, a aspersão favorece uma série de doenças, podendo provocar perdas significativas de produção e qualidade de frutos (Barbosa, 1997; Marouelli \& Silva, 2000).
Keywords: Lycopersicon esculentum, trickle, irrigation system, subsurface drip.
Estudos exploratórios realizados no Brasil por Silva \& Marouelli (1995), Silva et al. (1999) e Barbosa (1997) têm demonstrado o potencial do gotejamento para a irrigação do tomateiro industrial. 